\title{
Over expression of PTEN induces apoptosis and prevents cell proliferation in breast cancer cells
}

\author{
Jinfeng $\mathrm{Wu}^{1,2,3}$, Hong $\mathrm{GaO}^{4}$, Wanyu $\mathrm{Ge}^{1,2}$ and Jie $\mathrm{He}^{1,2,3} \square$ \\ 'Department of Pathology, The First Affiliated Hospital of USTC, Division of Life Sciences and Medicine, University of Science and Technology of \\ China, Hefei, Anhui, 230036, China; ${ }^{2}$ Intelligent Pathology Institute, Division of Life Sciences and Medicine, University of Science and Technology \\ of China, Hefei, Anhui, 230036, China; ${ }^{3}$ Department of Pathology, The First Affiliated Hospital of USTC, Division of life Sciences and Medicine, \\ University of Science and Technology of China, Hefei, Anhui, 230031, China; ${ }^{4}$ Department of Head And Neck Breast Surgery, The First Affiliated \\ Hospital of USTC, Division of Life Sciences and Medicine, University of Science and Technology of China, Hefei, Anhui, 230031, China
}

The phosphatase and tensin homolog (PTEN) is a tumor suppressor lipid phosphatase frequently mutated or deleted in breast cancer cells. Loss of PTEN is associated with aberrant activation of P13K/AKT signaling pathways, which are responsible for uncontrolled cell cycle, migration and prolonged survival. Therefore, stability and functional PTEN is essential for prevention of cancer growth and migration. In the present study, we have determined the effect of PTEN over expression in apoptosis induction and cell proliferation in breast cancer cells. We showed that PTEN over expression significantly declined the cell proliferation rate during logarithmic growth phase. Furthermore, the PTEN over expression leads to the activation of mitochondrial based intrinsic apoptosis pathways, which is confirmed by the activation and over expression of caspases 9 and caspases 3 . In addition, the number of apoptotic cells are significantly more in PTEN over expressed cells, where they showed more apoptotic bodies in AO-EtBr and Hoechst 33344 staining. Finally, PTEN over expressed cells showed decreased chemo resistance as chemotherapeutic drugs kill them efficiently. Therefore, our findings suggest that tumor suppressive effect of PTEN is crucial for cancer prevention and thus PTEN might be a potential target for anti-cancer drugs.

Key words: breast cancer, PTEN, apoptosis, P13K/AKT, cell proliferation

Received: 23 May, 2020; revised: 21 July, 2020; accepted: 28 August, 2020; available on-line: 17 December, 2020

ఐe-mail: hejie2005g@sina.com

Abbreviations: $\mathrm{AO}-\mathrm{EtBr}$, acridine orange/ethidium bromide; FAK, 5-FU, 5-fluorouracil; HER2, epidermal growth factors; PI3K, phosphatidylinositol-3-kinase; PTEN, phosphatase and tensin

\section{INTRODUCTION}

In world wide, breast cancer is the most common cancer and one of the leading cause for cancer associated death rate among women (Becker, 2015). Breast cancer is heterogeneous whose molecular features include activation of hormone receptors (oestrogen and progesterone), epidermal growth factors (HER2) mutations in BRCA gens. Breast cancer is curable when it is diagnosed at early stage non-metastatic condition and it also depends on the patient's age because the main risk factor of the breast cancer is the women age (Harbeck et al., 2019). Advanced stages at distant organ metastasis are usually treated with different treatment strategies for instance surgery, radiation therapy and systemic therapy such as endocrine therapy for hormone receptors, chem- otherapy and anti-HER2 therapy (Ataollahi et al., 2015; Carbognin et al., 2019; Harbeck et al., 2019). The ultimate goals of such treatment approaches are prolonged survival and improved quality of patient's life. Although recent developments of molecular approaches in cancer treatment and plethora of drugs designed, the enhanced proliferation rate and cancer cell migration are the major implications in the diagnosis and treatment of cancers. Considering this fact, researchers should get more insights in to the molecular biology and immunology to unravel the molecular characteristics cancer metastasis. Therefore, detailed study of molecular and signaling pathways involved in cancer progression is urging one to design and develop a right anti-cancer drug.

Phosphatase and tensin (PTEN) is a lipid and protein phosphatase, acts as a tumor suppressor involved in the regulation of cell cycle, cell growth and survival (Li et al., 1997; Steck et al., 1997; Li et al., 2015). The key function of PTEN is inhibiting or down regulating the phosphatidylinositol-3-kinase (PI3K)/protein kinase $\mathrm{B}$ (Akt) signaling, a pathway involved in various cellular processes such as cell proliferation, motility, invasion and survival (Li et al., 2015). Therefore, PTEN exhibits anti-oncogenic effect by attenuating P13K/AKT pathway through dephosphorylation of PIP3 (phosphatidylinositol (3,4,5)-trisphosphate) to PIP2 (phosphatidylinositol (4,5)-bisphosphate), and thus terminating the signals from growth factors for PI3K/AKT signal transduction pathways and activation of downstream targets ( $\mathrm{Lu}$ et al., 2016). However, it remains unclear whether PTEN can target any other cellular pathways other than P13K/ AKT. But it was reported that PTEN exert tumor suppressive effect by potentially inhibiting cell cycle progression, telomerase activity and as a consequence apoptosis was induced (Tian et al., 1999; Weng et al., 2001). Loss or mutated PTEN gene was frequently spotted at different stages of cancers such as several cancers such as breast, brain, prostate, lung, liver, colorectal and endometrium cancers ( $\mathrm{Li}$ et al., 1997; Li et al., 2016). Further, loss of PTEN expression and its function has been associated with aggressive tumor phenotypes accompanied with reduced apoptosis and enhanced cell proliferation. Therefore, we have investigated the effect of over expression of PTEN on apoptosis induction, cell proliferation and tumor invasion in breast cancer cells (MCF-7).

\section{MATERIAL AND METHODS}

Cell culturing. The breast cancer cell lines MCF-7 and MCF-7 over expressing PTEN were obtained from 
Anhui Provincial Cancer Hospital. Cells were cultured in DMEM with 10\% FBS and antibiotics such as penicillin and streptomycin. Cells were usually maintained in 5\% $\mathrm{CO}_{2}$ with humidified air at $37^{\circ} \mathrm{C}$. Cells were either cultured in Corning petri dished or T75 flask.

Plasmid transfection. Cells were cultured in 6 well plate for 24 hours in $5 \% \mathrm{CO}_{2}$ at $37^{\circ} \mathrm{C}$. For transfection, $8 \mu \mathrm{L}$ of either of the plasmids pCMV-PTEN (Addgene) or GFP-PTEN (Addgene) are mixed in OPtiMEM (Thermofishers) containing $6 \mu \mathrm{L}$ of transfection reagent Lipofectamine (Qiagen) and incubate at room temperature. After 20 minutes of incubation, the reaction mixture was added to the cells and incubated for either 24 or 48 hours.

Western blot. After 48 hours incubation, the both control and transfected cells were collected by centrifugation. $100 \mu \mathrm{L}$ of sample lysis buffer (from Bio$\mathrm{rad}$ ) was added to lyse the cell, which was performed on ice for 20 minutes. Samples were further heated to $100^{\circ} \mathrm{C}$ for $5 \mathrm{~min}$ and the cell lysates were allowed to pass through the columns (Qiagen). Finally, protein samples were eluted by centrifugation. Proteins were separated by $10 \%$ separation gel and transferred to polyvinylidene difluoride (PVDF) membrane. The membrane was incubated with primary antibodies (from cell signaling) such as PTEN (1:2000), P13K (1:2000); AKT (1:1000); caspase 3 (1:1000); caspases $9(1: 1000)$; BCL-2 (1:2000) and GAPDH (1:5000) followed by incubation with secondary antibody (mouse or rabbit) conjugated with HRP (cell signaling 1:5000). ECL chemiluminescent reagent kit (from Biorad) was to detect the protein band.

Fluorescence Microscopy. Transiently transfected cells were collected after 48 hours by centrifugation for 5 minutes at $200 \mathrm{~g}$ and washed with 3 times to remove the cell debris. Subsequently cells were subjected to Hoechst 33344 staining by adding $10 \mu \mathrm{L}$ of $10 \mathrm{mg} / \mathrm{L}$ of Hoechst 33344 dye to the cell suspension, incubated at dark for 30 minutes. Simultaneously, another portion of cells were subjected to $10 \mu \mathrm{L}$ of acridine orange/ethidium bromide $(\mathrm{AO} / \mathrm{EtBr})$ solution. The stained cells were dripped (approximately $10 \mu \mathrm{L}$ ) on slides ad covered by coverslips. The morphological features of apoptotic cells

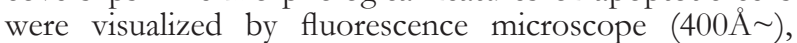
photographed and the acquired pictures were processed Adobe Photoshop software version 7.0.

Flow cytometry analysis. The apoptosis rate was measured by propidium iodide staining kit (Sigma-Aldrich). After 48 hours incubation of transient transfection with PTEN over expression cassette, cells were harvested and fixed in $70 \%$ ice-cold methanol. After overnight incubation with PI and RNase at a $37^{\circ} \mathrm{C}$ water bath in dark condition, cells were analyzed by flow cytometry and data were represented as a quantification graph showing percentage of apoptosis rate.

In vitro cell proliferation assay. Cells were seeded in a 96-well plate with a range of $1 \times 10^{6}$ cells/well (in triplicates) and the cell proliferation activity was measured every day for 7 days. 2-3 Hours before optical density measurement, cells were supplemented with CCK-8 solution $(10 \mu \mathrm{L})$ and incubated in $\mathrm{CO}_{2}$ incubator. Subsequently, optical density (OD) was determined at $490 \mathrm{~nm}$ was measured and the cell growth rate graph was made on the OD values and represented as a graph.

Chemoresistance assay. Cells were seeded in a 96well plate with a range of $1 \times 10^{6}$ cells/well (in triplicates). Followed by 24 hours incubation, docetaxel $(10 \mu \mathrm{M})$ and doxorubicin and 5-fluorouracil (5-FU) of final concentra- tion of $10 \mu \mathrm{g} / \mathrm{ml}$ was added to the cell cultures. After 48 hours, chemoresistance assay and the formula was evaluated as described previously (He et al., 2014).

Statistical analysis. The t-test was used to compare between the groups and between multi-groups were analyzed by a multi-factor $Q$-test. The values defined were $* P<0.05$ and $* * P<0.01$ significant.

\section{RESULTS}

\section{PTEN over expression inhibits cell proliferation via downregulating P13K/AKT signaling pathway}

It has been previously reported that over expression of PTEN contributes to cell cycle arrest by and induce apoptosis by suppressing the P13K/AKT pathway in hepatocellular carcinoma, renal carcinoma, breast cancer cells and glioma cells (Li et al., 2016; Lu et al., 2016). Therefore, we have investigated the protein expression levels of PTEN, P13K and AKT in MCF-7 breast cancer cells over expressing PTEN. As shown in the Fig. 1A, the reduced PTEN protein was observed in control cells and thus indicating the tumor suppressor PTEN protein level is modulated in cancer cells and thus leads to aberrant activation of $\mathrm{P} 13 \mathrm{~K}$ and AKT proteins. However, when we over express PTEN in breast cancer cells, the expression level of $\mathrm{P} 13 \mathrm{~K} / \mathrm{AK} \mathrm{T}$ pathways are significantly downregulated.

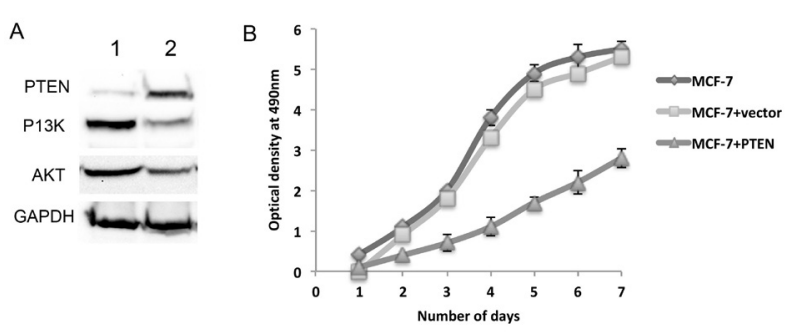

Figure 1. A. Western blot analysis for the expression pattern of PTEN, P13K and AKT. B. In vitro cell proliferation growth curve showing decreased growth rate of PTEN over expressing cells. Error bar is the standard deviation. ${ }^{*} P<0.05 ;{ }^{* *} P<0.01$.

Next we have analyzed the cell proliferation rate between the control and PTEN over expressed breast cancer cells. The cell growth curve (Fig. 1B) showing the logarithmic growth phase for the first four days after that the cell growth was gradually deceased. In these four days, the PTEN over expressed cells showed significantly compromised cell growth $(P<0.05)$ when compared to the controls. These results suggest that PTEN over expression suppressing the cell proliferation efficiently and that might be due to the cell cycle arrest (G1 phase) by inhibiting $\mathrm{P} 13 \mathrm{~K} / \mathrm{AKT}$ pathways as reported in the previous studies (Tamura et al., 1998; Weng et al., 1999; Ding et al., 2009; Gao et al., 2009; Lu et al., 2016).

\section{PTEN over expression induces apoptotic cell death}

We have examined the PTEN over expressed cells for apoptosis induction. Activation of caspases are the crucial event for the mitochondrial mediated intrinsic apoptotic pathways. Hence, we have analyzed the expression levels of caspases 9, caspases 3 and BCL- 

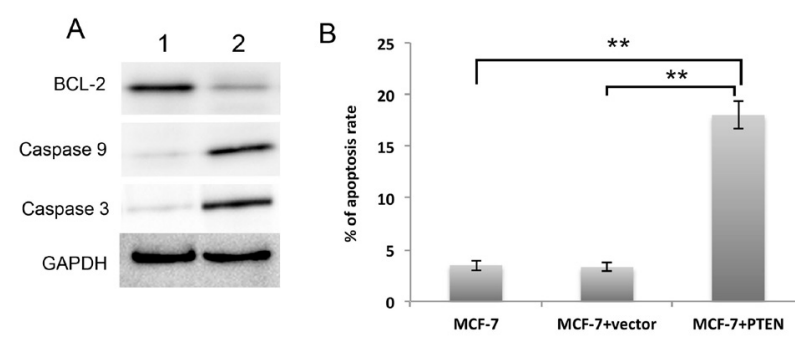

Figure 2. A. Western blot analysis showing the differential expression of apoptotic proteins in control and PTEN over expressed breast cancer cells. B. Quantitation graph made from flow cytometry analysis showing that rate of apoptosis are significantly higher in PTEN over expressed cells.

Error bar is the standard deviation. ${ }^{* *} P<0.01$.

2. By western blot analysis, we found that caspases 9 and caspase 3 are significantly enhanced in PTEN over expressed breast cancer cells. Simultaneously, the anti-apoptotic or pro-survival protein BCL-2 expression level is reduced (Fig. 2A). We have quantified the rate of apoptosis between the control and PTEN over expressed cells by flow cytometry. We found that significantly enhanced rate of apoptosis $(P<0.01)$ in the group over expressing PTEN (Fig. 2B). This data suggest that apoptosis is initiated in the PTEN over expressed cells. Accordingly, the post transfected cells were subjected to apoptosis evaluation by $\mathrm{AO}-\mathrm{EtBr}$ staining and Hoechst 33344 staining. By fluorescence microscopic analysis, we observed significantly more dead cells in PTEN over expressed groups, which emits red fluorescence confirming the apoptotic cells (Fig. 3A); whereas the live cells extrapolate green fluorescence. Further, the morphology of apoptotic cells is visualized as chromatin condensation and presence of bright blue pyknotic nucleus, which are not observed in control cells (Fig. 3B). Finally, our flow cytometry analysis revealed All these findings suggest that PTEN over expression efficiently induce apoptotic cell death in breast cancer cells and these data were consistent with previous findings in different cancers.

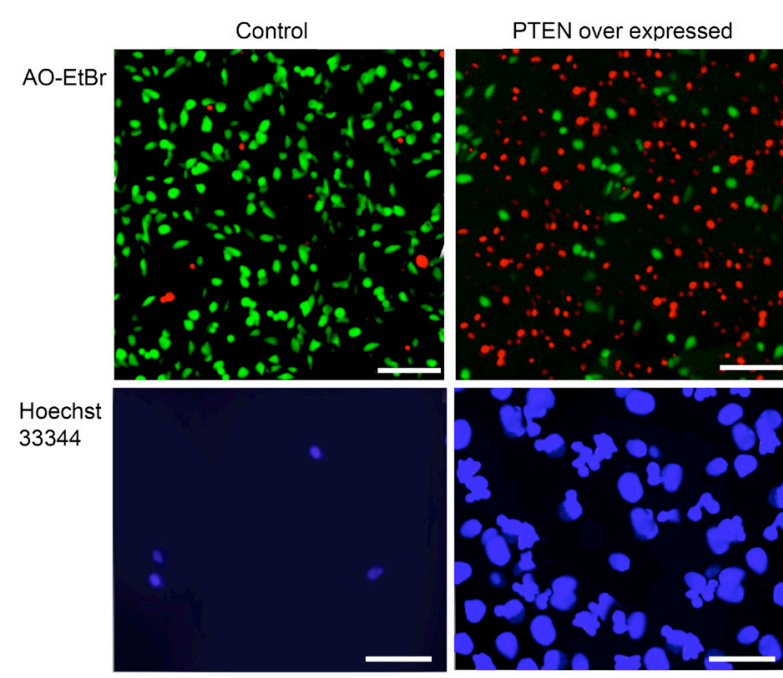

Figure 3. A. Fluorescence microscopic analysis of AO-EtBr staining showing the presence of more apoptotic cells, which infers red fluorescence, compared to control cells. B. Hoechst 33344 staining of PTEN over expressed cells showing more apoptotic features such as chromatin condensation and pyknotic nucleus in bright blue fluorescence.

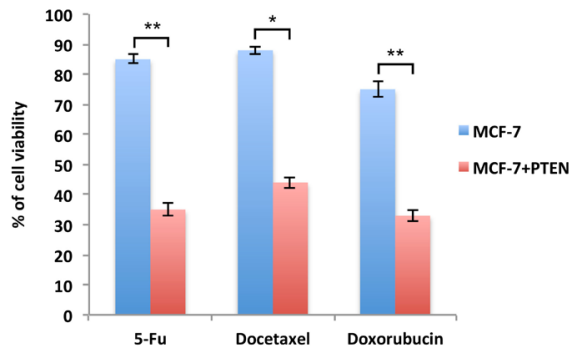

Figure 4. Quantification graph made from the chemoresistance assay displaying that PTEN over expressed cells are more susceptible and their viability was reduced significantly. Error bar is the standard deviation. ${ }^{*} P<0.05$; ${ }^{* *} P<0.01$.

\section{PTEN over expression contributes increased sensitivity to chemotherapy drugs}

Major hurdles of the current chemotherapy strategies are the multi-drug resistance and tumor regrowth of cancer cells owing to the over expression of ABC transporter proteins. We hypothesize that reduced expression of tumor suppressor gene PTEN might also contribute to chemotherapy resistance. Therefore, we have performed chemotherapy resistance assay to determine the effect of PTEN over expression on cell viability for 5-FU, docetaxel and doxorubicin. Figure 4 showing that PTEN over expressed cells showed significantly increased sensitivity towards the above-mentioned drugs, when compared to controls. Therefore, the increased sensitivity towards chemotherapeutic drugs leads to apoptosis induction and cell death.

\section{DISCUSSION}

Studies have been demonstrated that PTEN can exert tumor suppressive effect by inducing apoptosis, inhibiting tumor invasion and maintaining genome integrity (Zhao et al., 2013). The PTEN mediated tumor suppression can be achieved by attenuation of the PI3K/ AKT signaling pathway and its downstream targets such as FRAP/mTOR, FAK and APK (Ohta et al., 2015). Therefore, for normal physiological processes such as regulated cell cycle, cell proliferation, differentiation, apoptosis and metabolism PTEN gene function is essential. However, PTEN was found to be mutated or loss its function frequently and as a consequence P13K/ AKT pathway is aberrantly activated in cancer cells (Carbognin et al., 2019).

In the present study, the breast cancer cells showed reduced expression of PTEN and enhanced regulation of $\mathrm{P} 13 \mathrm{~K} / \mathrm{AK} \mathrm{T}$ signaling pathways, which are crucial for increased cell proliferation, malignant transformation and migration. In the mean time, when we over express the PTEN in breast cancer cells, the P13K/ AKT pathway is down regulated, which is confirmed by our western blot analysis. This finding is consistent with the previous reports where they showed mutated or deleted PTEN results in excessive activation of P13K signal leads to hyper cell proliferation (Yan et al., 2015). However, when we complement the PTEN function in breast cancer cells, the rate of proliferation was significantly reduced when compared to the control cells. PTEN inhibits P13K signaling and its downstream targets by dephosphorylation of PIP3 to PIP2 so that AKT binding is interrupted. Consequently, the PTEN induce cell cycle arrest at G1 phase and ultimately the induction of apoptosis takes place (Tamura et al., 1998; Ding 
et al., 2009; Gao et al., 2009). Studies in MCF-7 cell line observed stimulation of apoptosis and cell cycle arrest in G1 phase upon over expression of PTEN under serum free conditions (Lu et al., 1999; Zhu et al., 2001; Li et al., 2007; Yan et al., 2015). In addition, the focal adhesion kinase phosphorylation also regulated by PTEN which in turn prevent cell adhesion, migration and apoptosis ( $\mathrm{Lu}$ et al., 2016).

In our study, we have also examined the impact of PTEN over expression in apoptosis induction in breast cancer cells. We have shown the correlation between the number of apoptotic cells by flow cytometry/staining (PI, Hoechst 33344 and $\mathrm{AO}-\mathrm{EtBr}$ ) and expression of caspases involved in mitochondria dependent apoptosis. Our study further revealed that PTEN over expression cause down regulation of pro-survival protein BCL-2. As a result, mitochondrial membrane potential is distorted and releases the cytochrome $c$. Several studies has been reported that released cytochrome $\mathrm{c}$ involved in the activation of caspases 9, which binds to the Apoptotic protease activating factor 1 (APAF-1). In turn, caspases 3 gets activated and thus cause apoptotic cell death (Weng et al., 2009; McIlwain et al., 2013; Campbell et al., 2018). Consequently, in our study we have also estimated the expression level of cytochrome $c$ by western blot analysis. We found that cytochrome $c$ level is enhanced in the PTEN over expressed MCF-7 cells (not shown). Therefore, PTEN over expressed breast cancer cells showed enhanced mitochondrial dependent intrinsic apoptosis, which is confirmed by caspases over expression and the persistence apoptotic features. Our findings are in agreed with previously reports, which stated that over expression of PTEN is responsible for the induction of apoptosis (Lu et al., 1999; Weng et al., 2001; Li et al., 2007; Hemmings et al., 2012; Carbognin et al., 2019).

The interesting finding in our study is PTEN over expression breast cancer cells are more susceptible towards chemotherapeutic drugs such as 5-FU, docetaxel and doxorubicin, which killed cells effectively. This suggests that PTEN over expressed can efficiently uptake the drugs leads to induction of apoptosis and thus cell viability is deceased. However, findings from our present study are preliminary and the lack of animal models and in vivo experiments are the limitations. In future, detailed research work will be carried by performing more in vivo experiments by using animal models and different cancer cell lines as well.

Meanwhile, Liu and others (Liu et al., 2012) showed that PTEN over expression combined with the docetaxel are effective adjuvant therapy for liver cancer. Similarly, it has been demonstrated in MCF-7 cells that PTEN plays an essential role in the etoposide induced check point activation and cell cycle arrest (Zhang et al., 2016). It is well known that chemoresistance property of the cancer cell is due to the over expression of ATPase binding cassette (ABC) transporter proteins which efflux the therapeutic drugs efficiently out of the cells (He et al., 2014; Wang et al., 2015). Therefore, further detailed investigation is requited to elucidate the role of PTEN in inhibiting $\mathrm{ABC}$ proteins expression and efficient drug uptake will provide more molecular insights and that would be clinically significant for the effective cancer treatment.

In summary, our finding suggests that over expression of PTEN attenuate the cell growth and induce apoptosis in breast cancer cells. Therefore, the tumor suppression activity exerted by PTEN is crucial for the prevention of cancer and therefore PTEN might be potential target for anti-cancer therapy.

\section{Conflicts of interest}

All authors declared no conflict of interest for this manuscript.

\section{REFERENCES}

Ataollahi MR, Sharifi J, Paknahad MR, Paknahad A (2015) Breast cancer and associated factors: a review. J Med Life 4: 6

Becker S (2015) A historic and scientific review of breast cancer: The next global healthcare challenge. Int J Gynecol Obstetrics 131: S36-S39. https://doi.org/10.1016/j.ijgo.2015.03.015

Campbell KJ, Tait SW (2018) Targeting BCL-2 regulated apoptosis in cancer. Open Biol 8: 180002. https://doi.org/10.1098/rsob.180002

Carbognin L, Miglietta F, Paris I, Dieci MV (2019) Prognostic and predictive implications of PTEN in breast cancer: unfulfilled promises but intriguing perspectives. Cancers 11: 1401. https://doi. org/10.3390/cancers11091401

Ding J, Ning B, Gong W, Wen W, Wu K, Liang J, He G, Huang S, Sun W, Han T, Huang L (2009) Cyclin D1 induction by benzo [a] pyrene-7, 8-diol-9, 10-epoxide via the phosphatidylinositol 3-kinase/ Akt/MAPK-and p70s6k-dependent pathway promotes cell transformation and tumorigenesis. J Biol Chem 284: 33311-3319. https:// doi.org/10.1074/ibc.M109.046417

Gao Q, Ye F, Xia X, Xing H, Lu Y, Zhou J, Ma D (2009) Correlation between PTEN expression and PI $3 \mathrm{~K} / \mathrm{Akt}$ signal pathway in endometrial carcinoma. Med Sci 29: 59-63. https://doi.org/10.1007/ s11596-009-0112-6

Harbeck N, Penault-Llorca F, Cortes J, Gnant M, Poortmans P, Ruddy K, Tsang J, Cardoso F (2019) Breast cancer. Nat Rev Dis Primers 5: 66. https://doi.org/10.1038/s41572-019-0111-2

He QZ, Luo XZ, Wang K Zhou Q, Ao H, Yang Y, Li SX, Li Y, Zhu HT, Duan T (2014) Isolation and characterization of cancer stem cells from high-grade serous ovarian carcinomas. Cell Physiol Biochem 33: 173-184. https://doi.org/10.1159/000356660

Hemmings BA, Restuccia DF (2012) Pi3k-pkb/akt pathway. Cold Spring Harbor Perspectives Biol 4: a011189. https://doi.org/10.1101/cshperspect.a011189

Li DM, Sun H (1997) TEP Encoded by a candidate tumor suppressor locus, is a novel protein tyrosine phosphatase regulated by transforming growth factor beta. Cancer Res 57: 2124-2129

Li J, Yen C, Liaw D, Podsypanina K, Bose S, Wang SI, Puc J, Miliaresis C, Rodgers L, McCombie R, Bigner SH (1997) PTEN, a putative protein tyrosine phosphatase gene mutated in human brain, breast, and prostate cancer. Science 275: 1943-1947. https://doi. org/10.1126/science.275.5308.1943

Li X, Lin G, Wu B, Zhou X, Zhou K (2007) Overexpression of PTEN induces cell growth arrest and apoptosis in human breast cancer ZR-75-1 cells. Acta Biochim Biophys Sinica 39: 745-750. https://doi.org/10.1111/j.1745-7270.2007.00337.x

Li MF, Guan H, Zhang DD (2016) Effect of overexpression of PTEN on apoptosis of liver cancer cells. Genet Mol Res 15: 10-4238. https://doi.org/10.4238/gmr.15028120

Liu Z, Li J, Li J, Huang J, Ke F, Qi Q, Jiang X, Zhong Z (2012) Mannan-modified Ad5-PTEN treatment combined with docetaxel improves the therapeutic effect in $\mathrm{H} 22$ tumor-bearing mice. Int J Nanomed 7: 5039. https://doi.org/10.2147/IJN.S34022

Lu Y, Lin YZ, LaPushin R, Cuevas B, Fang X, Yu SX, Davies MA, Khan H, Furui T, Mao M, Zinner R (1998) The PTEN/MMAC1/ TEP tumor suppressor gene decreases cell growth and induces apoptosis and anoikis in breast cancer cells. Oncogene 18: 7034-7045. https://doi.org/10.1038/sj.onc.1203183

Lu XX, Cao LY, Chen X, Xiao J, Zou Y, Chen Q (2016) PTEN inhibits cell proliferation, promotes cell apoptosis, and induces cell cycle arrest via downregulating the PI3K/AKT/hTERT pathway in lung adenocarcinoma A549 cells. BioMed Res Int 2016: 2016. https:// doi.org/ 10.1155/2016/2476842

McIlwain DR, Berger T, Mak TW (2013) Caspase functions in cell death and disease. Cold Spring Harbor Perspectives Biol 5: a008656. https://doi.org/10.1101/cshperspect.a026716

Ohta K, Hoshino H, Wang J, Ono S, Iida Y, Hata K, Huang SK, Colquhoun S, Hoon DS (2015) MicroRNA-93 activates c-Met/ PI3K/Akt pathway activity in hepatocellular carcinoma by directly inhibiting PTEN and CDKN1A. Oncotarget 6: 3211. https://doi. org/10.18632/oncotarget.3085

Steck PA, Pershouse MA, Jasser SA, Yung WA, Lin H, Ligon AH, Langford LA, Baumgard ML, Hattier T, Davis T, Frye C (1997) Identification of a candidate tumour suppressor gene, MMAC1, at chromosome 10q23. 3 that is mutated in multiple advanced cancers. Nat Genet 15: 356-362. https://doi.org/10.1038/ng0497-356

Tamura M, Gu J, Matsumoto K, Aota SI, Parsons R, Yamada KM (1998) Inhibition of cell migration, spreading, and focal adhesions by tumor suppressor PTEN. Science 280: 1614-1617. https://doi. org/10.1126/science.280.5369.1614 
Tian XX, Pang JC, To SS, Ng HK (1999) Restoration of wild-type PTEN expression leads to apoptosis, induces differentiation, and reduces telomerase activity in human glioma cells. J Neuropath Exp Neurol 58: 472-479. https://doi.org/10.1097/00005072-19990500000006

Wang C, Youle RJ (2009) The role of mitochondria in apoptosis. Ann Rev Genet 43: 95-118. https://doi.org/10.5483/bmbrep.2008.41.1.011

Wang M, Wang Y, Zhong J (2015) Side population cells and drug resistance in breast cancer. Mol Med Rep 11: 4297-4302. https://doi. org $/ 10.3892 / \mathrm{mmr} .2015 .3291$

Weng LP, Smith WM, Dahia PL, Ziebold U, Gil E, Lees JA, Eng C (1999) PTEN suppresses breast cancer cell growth by phosphatase activity-dependent G1 arrest followed by cell death. Cancer Res 59: 5808-5814

Weng LP, Brown JL, Eng C (2001) PTEN induces apoptosis and cell cycle arrest through phosphoinositol-3-kinase/Akt-dependent and independent pathways. Human Mol Genet 10: 237-242. https://doi. org/10.1093/hmg/10.3.237

Yan SY, Chen MM, Li GM, Wang YQ, Fan JG (2015) MiR-32 induces cell proliferation, migration, and invasion in hepatocellular carcinoma by targeting PTEN. Tumor Biol 36: 4747-4755. https://doi.org/ 10.1007/s13277-015-3124-9

Zhang R, Zhu L, Zhang L, Xu A, Li Z, Xu Y, He P, Wu M, Wei F, Wang C (2016) PTEN enhances G2/M arrest in etoposide-treated MCF-7 cells through activation of the ATM pathway. Oncol Rep 35: 2707-2714. https://doi.org/10.3892/or.2016.4674

Zhao B, Zhu Y, Cui K, Gao J, Yu F, Chen L, Li S (2013) Expression and significance of PTEN and miR-92 in hepatocellular carcinoma. Mol Med Rep 7: 1413-1416. https://doi.org/10.3892/mmr.2013.1406 Zhu X, Kwon CH, Schlosshauer PW, Ellenson LH, Baker SJ (2011) PTEN induces G1 cell cycle arrest and decreases cyclin D3 levels in endometrial carcinoma cells. Cancer Res 61: 4569-4575 